\title{
Hyers-Ulam Stability of Fractional Nabla Difference Equations
}

\author{
Jagan Mohan Jonnalagadda \\ Department of Mathematics, Birla Institute of Technology and Science Pilani, Hyderabad, Telangana 500078, India
}

Correspondence should be addressed to Jagan Mohan Jonnalagadda; j.jaganmohan@hotmail.com

Received 21 July 2016; Accepted 28 August 2016

Academic Editor: Dong Ye

Copyright (C) 2016 Jagan Mohan Jonnalagadda. This is an open access article distributed under the Creative Commons Attribution License, which permits unrestricted use, distribution, and reproduction in any medium, provided the original work is properly cited.

We investigate the Hyers-Ulam stability, the generalized Hyers-Ulam stability, and the $F_{\alpha}$-Hyers-Ulam stability of a linear fractional nabla difference equation using discrete Laplace transform. We provide a few examples to illustrate the applicability of established results.

\section{Introduction}

Ulam [1] posed the following problem on the stability of functional equations in 1940.

Ulam's Problem (see [1]). Given a group $G_{1}$, a metric group $\left(G_{2}, d\right)$, and a positive number $\varepsilon$, does there exist $\delta>0$ such that if a mapping $f: G_{1} \rightarrow G_{2}$ satisfies the inequality

$$
d(f(x y), f(x), f(y))<\delta
$$

for all $x, y \in G_{1}$, then there exists a homomorphism $T: G_{1} \rightarrow$ $G_{2}$ such that

$$
d(f(x), T(x))<\delta,
$$

for all $x, y \in G_{1}$ ?

Hyers [2] solved the problem for additive functions defined on Banach spaces in 1941 as follows.

Hyer's Theorem (see [2]). Let $E_{1}$ be a normed vector space and $E_{2}$ a Banach space and suppose that the mapping $f: E_{1} \rightarrow E_{2}$ satisfies the inequality

$$
\|f(x+y)-f(x)-f(y)\| \leq \varepsilon,
$$

for all $x, y \in E_{1}$, where $\varepsilon>0$ is a constant. Then the limit

$$
g(x)=\lim _{n} 2^{-n} f\left(2^{n} x\right)
$$

exists for each $x \in E_{1}$ and $g$ is the unique additive mapping satisfying

$$
\|f(x)-g(x)\| \leq \varepsilon,
$$

for all $x \in E_{1}$.

Rassias [3] provided a generalization of the Hyers theorem for linear mappings. Later, many mathematicians have extended Ulam's problem in different directions. Recently, a generalization of Ulam's problem on the stability of differential equations was proposed.

Let $X$ be a normed space and $I$ be an open interval. The differential equation $F\left(t, u, u^{\prime}, u^{\prime \prime}, \ldots, u^{(n)}\right)=0$ is HyersUlam stable, if, for given $\varepsilon>0$ and a function $v: I \rightarrow X$ such that $\left\|F\left(t, v, v^{\prime}, v^{\prime \prime}, \ldots, v^{(n)}\right)\right\| \leq \varepsilon$, there exists a solution $u: I \rightarrow X$ of the differential equation such that $\| u(t)-$ $v(t) \| \leq K(\varepsilon)$ for any $t \in I$, where $K(\varepsilon)$ is an expression of $\varepsilon$ only. If the above statement is also true when we replace $\varepsilon$ and $K(\varepsilon)$ by $\phi(t)$ and $\psi(t)$, where $\phi, \psi: I \rightarrow[0, \infty)$ are functions not depending on $v$ and $u$ explicitly, then we say that the corresponding differential equation has the generalized Hyers-Ulam stability. For a detailed discussion on the Hyers-Ulam stability, refer to $[4,5]$.

Recently, Rezaei et al. [6] obtained the Hyers-Ulam stability of a linear differential equation using Laplace transforms. Motivated by this article, Wang and $\mathrm{Xu}[7,8]$ and Wang and Li [9] investigated the same for a class of linear fractional differential equations involving both Riemann-Liouville and 
Caputo type fractional derivatives. In this article, we extend this study to linear fractional nabla difference equations.

\section{Preliminaries}

Throughout this article, we use the following notation, definitions, and known results of fractional nabla calculus [10]: Denote the set of all real numbers and complex numbers by $\mathbb{R}$ and $\mathbb{C}$, respectively. Define $\mathbb{N}_{a}=\{a, a+1, a+2, \ldots\}$ for any $a \in \mathbb{R}$. Assume that empty sums and products are taken to be 0 and 1 , respectively.

Definition 1 (rising factorial function). For any $\alpha \in \mathbb{R}, t \in$ $\mathbb{R} \backslash\{\ldots,-2,-1,0\}$ such that $(t+\alpha) \in \mathbb{R} \backslash\{\ldots,-2,-1,0\}$, the rising factorial function is defined by

$$
t^{\bar{\alpha}}=\frac{\Gamma(t+\alpha)}{\Gamma(t)}, \quad 0^{\bar{\alpha}}=0 .
$$

Definition 2. Let $u: \mathbb{N}_{a} \rightarrow \mathbb{R}$ and $0<\alpha<1$.

(1) (Fractional nabla sum) [11]: the $\alpha$ th-order nabla sum of $u$ is given by

$$
\left(\nabla_{a}^{-\alpha} u\right)(t)=\frac{1}{\Gamma(\alpha)} \sum_{s=a}^{t}(t-s+1)^{\overline{\alpha-1}} u(s), \quad t \in \mathbb{N}_{a}
$$

(2) (R-L fractional nabla difference) [11]: the $\alpha$ th-order nabla difference of $u$ is given by

$$
\left(\nabla_{a}^{\alpha} u\right)(t)=\frac{1}{\Gamma(-\alpha)} \sum_{s=a}^{t}(t-s+1)^{\overline{-\alpha-1}} u(s), \quad t \in \mathbb{N}_{a+1}
$$

(3) (Caputo fractional nabla difference) [11]: the $\alpha$ thorder nabla difference of $u$ is given by

$$
\left(\nabla_{a *}^{\alpha} u\right)(t)=\left(\nabla_{a}^{\alpha} u\right)(t)-\frac{(t-a+1)^{\overline{-\alpha}}}{\Gamma(1-\alpha)} u(a),{ }_{t \in \mathbb{N}_{a+1}}
$$

Nagai [12] and Atici and Eloe [13] defined the one- and two-parameter Mittag-Leffler functions of fractional nabla calculus as follows.

Definition 3 (see $[12,13])$. The one- and two-parameter nabla Mittag-Leffler functions are defined by

$$
\begin{gathered}
F_{\alpha}\left(\lambda,(t-a)^{\bar{\alpha}}\right)=\sum_{k=0}^{\infty} \frac{\lambda^{k}(t-a)^{\overline{k \alpha}}}{\Gamma(k \alpha+1)}, \\
F_{\alpha, \beta}\left(\lambda,(t-a)^{\bar{\alpha}}\right)=\sum_{k=0}^{\infty} \frac{\lambda^{k}(t-a)^{\overline{k \alpha}}}{\Gamma(k \alpha+\beta)},
\end{gathered}
$$

where $\alpha, \beta>0,|\lambda|<1$, and $t \in \mathbb{N}_{a}$.

Estimates of nabla Mittag-Leffler functions are provided in Lemma 4.
Lemma 4. Let $0<\alpha, \beta<1$. The functions $F_{\alpha}$ and $F_{\alpha, \alpha}$ are nonnegative and for any $0<\lambda<1$ and $t \in \mathbb{N}_{0}$,

$$
\begin{gathered}
F_{\alpha}\left(-\lambda, t^{\bar{\alpha}}\right) \leq 1, \\
F_{\alpha, \alpha}\left(-\lambda, t^{\bar{\alpha}}\right) \leq \frac{1}{\Gamma(\alpha)} .
\end{gathered}
$$

Definition 5 (see [13]). Let $u: \mathbb{N}_{a} \rightarrow \mathbb{R}$. The $N$-transform of $u$ is defined by

$$
N_{a}[u(t)]=\sum_{j=a}^{\infty} u(j)(1-z)^{j-1},
$$

for each $z \in \mathbb{C}$ for which the series converges.

Definition 6 (see [13]). Let $u, v: \mathbb{N}_{a} \rightarrow \mathbb{R}$. The convolution of $u$ and $v$ is defined by

$$
\left(u *_{a} v\right)(t)=\sum_{s=a}^{t} u(t+a-\rho(s)) v(s) .
$$

Atici and Eloe [13] developed the following properties of $N$-transforms.

Theorem 7 (see [13]). Assume that the following functions are well defined:

(1) $N_{a}\left[\left(u *{ }_{a} v\right)(t)\right]=N_{1}[u(t+a)] N_{a}[v(t)]$.

(2) $N_{a}\left[(t-a+1)^{\bar{\alpha}}\right]=(1-z)^{a-1}\left(\Gamma(\alpha+1) / z^{\alpha+1}\right), \alpha \epsilon$ $\mathbb{R} \backslash\{\ldots,-3,-2,-1\}$.

(3) $N_{a}\left[\left(\nabla_{a}^{\alpha} u\right)(t)\right]=z^{\alpha} N_{a}[u(t)], 0<\alpha<1$.

(4) $N_{a+1}\left[\left(\nabla_{a}^{\alpha} u\right)(t)\right]=z^{\alpha} N_{a}[u(t)]-(1-z)^{a-1} u(a), 0<$ $\alpha<1$.

(5) $N_{a}\left[(t-a+1)^{\overline{\alpha-1}} F_{\alpha, \alpha}\left(\lambda,(t-a+\alpha)^{\bar{\alpha}}\right)\right]=(1-z)^{a-1} /\left(z^{\alpha}-\right.$ $\lambda)$.

\section{Main Results}

The main purpose of this section is to discuss the Hyers-Ulam stability of the following difference equation:

$$
\left(\nabla_{0}^{\alpha} u\right)(t)+\lambda u(t)=f(t), \quad 0<\alpha<1, \lambda \geq 0,
$$

where $u, f: \mathbb{N}_{0} \rightarrow \mathbb{R}$ and $\lambda$ is a constant.

Let $v: \mathbb{N}_{0} \rightarrow \mathbb{R}$ and $w(t)=\left(\nabla_{0}^{\alpha} v\right)(t)+\lambda v(t)-f(t)$ for $t \in \mathbb{N}_{0}$. Using Theorem 7 , we have

$$
\begin{aligned}
N_{1}[w(t)] \\
=\left[\left(z^{\alpha}+\lambda\right) N_{0}[v(t)]-(1+\lambda)\left(\frac{1}{1-z}\right) v(0)\right] \\
\quad-\left[N_{0}[f(t)]-\left(\frac{1}{1-z}\right) f(0)\right] \\
=\left(z^{\alpha}+\lambda\right) N_{0}[v(t)]-N_{0}[f(t)] \\
\quad-\left(\frac{1}{1-z}\right)[(1+\lambda) v(0)-f(0)],
\end{aligned}
$$


which implies

$$
\begin{gathered}
N_{0}[v(t)]=\left(z^{\alpha}+\lambda\right)^{-1}\left[N_{1}[w(t)]+N_{0}[f(t)]\right. \\
\left.+\left(\frac{1}{1-z}\right)[(1+\lambda) v(0)-f(0)]\right] .
\end{gathered}
$$

Set

$$
\begin{aligned}
u(t) & \\
= & v(0)(1+\lambda)(t+1)^{\overline{\alpha-1}} F_{\alpha, \alpha}\left(-\lambda,(t+\alpha)^{\bar{\alpha}}\right) \\
& +\sum_{s=1}^{t}(t-s+1)^{\overline{\alpha-1}} F_{\alpha, \alpha}\left(-\lambda,(t-s+\alpha)^{\bar{\alpha}}\right) f(s) .
\end{aligned}
$$

Clearly $u(0)=v(0)$. Applying the $N_{0}$ transform on both sides of (17), we get

$$
\begin{aligned}
N_{0} & {[u(t)]=[(1+\lambda) v(0)-f(0)] N_{0}\left[(t+1)^{\overline{\alpha-1}}\right.} \\
\cdot & \left.F_{\alpha, \alpha}\left(-\lambda,(t+\alpha)^{\bar{\alpha}}\right)\right]+N_{0}\left[\sum_{s=0}^{t}(t-s+1)^{\overline{\alpha-1}}\right. \\
& \left.\cdot F_{\alpha, \alpha}\left(-\lambda,(t-s+\alpha)^{\bar{\alpha}}\right) f(s)\right]=[(1+\lambda) v(0) \\
& -f(0)] N_{0}\left[(t+1)^{\overline{\alpha-1}} F_{\alpha, \alpha}\left(-\lambda,(t+\alpha)^{\bar{\alpha}}\right)\right] \\
& +N_{0}\left[t^{\overline{\alpha-1}} F_{\alpha, \alpha}\left(-\lambda,(t-1+\alpha)^{\bar{\alpha}}\right) *_{0} f(t)\right] \\
& =\left(\frac{(1+\lambda) v(0)-f(0)}{1-z}\right)\left(z^{\alpha}+\lambda\right)^{-1} \\
& +N_{1}\left[t^{\overline{\alpha-1}} F_{\alpha, \alpha}\left(-\lambda,(t-1+\alpha)^{\bar{\alpha}}\right)\right] N_{0}[f(t)] \\
& =\left(\frac{(1+\lambda) v(0)-f(0)}{1-z}\right)\left(z^{\alpha}+\lambda\right)^{-1}+\left(z^{\alpha}+\lambda\right)^{-1} \\
& \cdot N_{0}[f(t)],
\end{aligned}
$$

which implies

$$
\begin{aligned}
& \left(z^{\alpha}+\lambda\right) N_{0}[u(t)]=\left(\frac{(1+\lambda) v(0)-f(0)}{1-z}\right) \\
& +N_{0}[f(t)] \\
& \text { or }\left[z^{\alpha} N_{0}[u(t)]-\left(\frac{1}{1-z}\right) u(0)\right] \\
& +\lambda\left[N_{0}[u(t)]-\left(\frac{1}{1-z}\right) u(0)\right] \\
& =\left[N_{0}[f(t)]-\left(\frac{1}{1-z}\right) f(0)\right] \\
& \text { or } N_{1}\left[\left(\nabla_{0}^{\alpha} u\right)(t)+\lambda u(t)\right]=N_{1}[f(t)] \text {. }
\end{aligned}
$$

Since $N_{1}$ is one-to-one, it follows that $\left(\nabla_{0}^{\alpha} u\right)(t)+\lambda u(t)=f(t)$, so $u(t)$ is a solution of (14). From (16) and (18), we get

$$
\begin{aligned}
N_{0} & {[u(t)]-N_{0}[v(t)]=\left(z^{\alpha}+\lambda\right)^{-1} N_{1}[w(t)]=\left(z^{\alpha}\right.} \\
+ & \lambda)^{-1}\left[N_{0}[w(t)]-\left(\frac{1}{1-z}\right) w(0)\right] \\
& =N_{1}\left[t^{\overline{\alpha-1}} F_{\alpha, \alpha}\left(-\lambda,(t-1+\alpha)^{\bar{\alpha}}\right)\right] N_{0}[w(t)] \\
& -w(0) N_{0}\left[(t+1)^{\overline{\alpha-1}} F_{\alpha, \alpha}\left(-\lambda,(t+\alpha)^{\bar{\alpha}}\right)\right] \\
& =N_{0}\left[t^{\overline{\alpha-1}} F_{\alpha, \alpha}\left(-\lambda,(t-1+\alpha)^{\bar{\alpha}}\right) *_{0} w(t)\right]-w(0) \\
& =N_{0}\left[(t+1)^{\overline{\alpha-1}} F_{\alpha, \alpha}\left(-\lambda,(t+\alpha)^{\bar{\alpha}}\right)\right] \\
& =N_{0}\left[\sum_{s=1}^{t}(t-s+1)^{\overline{\alpha-1}} F_{\alpha, \alpha}\left(-\lambda,(t-s+\alpha)^{\bar{\alpha}}\right) w(s)\right] .
\end{aligned}
$$

Since $N_{0}$ is one-to-one, it follows that

$$
\begin{aligned}
u(t) & -v(t) \\
& =\sum_{s=1}^{t}(t-s+1)^{\overline{\alpha-1}} F_{\alpha, \alpha}\left(-\lambda,(t-s+\alpha)^{\bar{\alpha}}\right) w(s) .
\end{aligned}
$$

First, we establish the generalized Hyers-Ulam stability of (14) as follows.

Theorem 8. Let $v: \mathbb{N}_{0} \rightarrow \mathbb{R}$ and $\phi: \mathbb{N}_{0} \rightarrow[0, \infty)$. If

$$
\left|\left(\nabla_{0}^{\alpha} v\right)(t)+\lambda v(t)-f(t)\right| \leq \phi(t), \quad t \in \mathbb{N}_{0},
$$

then, there exists a solution $u: \mathbb{N}_{0} \rightarrow \mathbb{R}$ of (14) and $\psi: \mathbb{N}_{0} \rightarrow$ $[0, \infty)$ such that

$$
|u(t)-v(t)| \leq \psi(t), \quad t \in \mathbb{N}_{0} .
$$

Proof. Using (21) and Lemma 4, we have

$$
\begin{aligned}
& |u(t)-v(t)| \\
& \quad \leq \sum_{s=1}^{t}(t-s+1)^{\overline{\alpha-1}} F_{\alpha, \alpha}\left(-\lambda,(t-s+\alpha)^{\bar{\alpha}}\right)|w(s)| \\
& \quad \leq \frac{1}{\Gamma(\alpha)} \sum_{s=1}^{t}(t-s+1)^{\overline{\alpha-1}} \phi(s)=\left(\nabla_{1}^{-\alpha} \phi\right)(t) \\
& \quad=\psi(t) .
\end{aligned}
$$

Now, consider a particular case of Theorem 8 which we define as the $F_{\alpha}$-Hyers-Ulam stability of (14).

Corollary 9. Let $\varepsilon>0,0<\eta<1$, and $v: \mathbb{N}_{0} \rightarrow \mathbb{R}$. If

$$
\left|\left(\nabla_{0}^{\alpha} v\right)(t)+\lambda v(t)-f(t)\right| \leq \varepsilon F_{\alpha}\left(\eta, t^{\bar{\alpha}}\right), \quad t \in \mathbb{N}_{0},
$$

then, there exists a solution $u: \mathbb{N}_{0} \rightarrow \mathbb{R}$ of (14) such that

$$
|u(t)-v(t)| \leq K(\varepsilon) F_{\alpha}\left(\eta, t^{\bar{\alpha}}\right), \quad t \in \mathbb{N}_{0},
$$


where

$$
K(\varepsilon)=\frac{\varepsilon}{\eta} .
$$

Next, we investigate the Hyers-Ulam stability of (14).

Theorem 10. Let $\varepsilon>0$ and $v: \mathbb{N}_{0} \rightarrow \mathbb{R}$. If

$$
\left|\left(\nabla_{0}^{\alpha} v\right)(t)+\lambda v(t)-f(t)\right| \leq \varepsilon, \quad t \in \mathbb{N}_{0},
$$

there exists a solution $u: \mathbb{N}_{0} \rightarrow \mathbb{R}$ of (14) such that

$$
|u(t)-v(t)| \leq K(\varepsilon), \quad t \in \mathbb{N}_{0},
$$

where

$$
K(\varepsilon)=\frac{\varepsilon}{\lambda} .
$$

Proof. Using (21) and Lemma 4, we have

$$
\begin{aligned}
\mid u(t) & -v(t) \mid \\
& \leq \sum_{s=0}^{t}(t-s+1)^{\overline{\alpha-1}} F_{\alpha, \alpha}\left(-\lambda,(t-s+\alpha)^{\bar{\alpha}}\right)|w(s)| \\
& \leq \varepsilon \sum_{s=0}^{t}(t-s+1)^{\overline{\alpha-1}} F_{\alpha, \alpha}\left(-\lambda,(t-s+\alpha)^{\bar{\alpha}}\right) \\
& =\varepsilon \sum_{s=0}^{t}(t-s+1)^{\overline{\alpha-1}} \sum_{k=0}^{\infty} \frac{(-\lambda)^{k}(t-s+\alpha)^{\overline{\alpha k}}}{\Gamma(\alpha k+\alpha)} \\
& =\varepsilon \sum_{k=0}^{\infty} \frac{(-\lambda)^{k}}{\Gamma(\alpha k+\alpha)} \sum_{s=0}^{t}(t-s+1)^{\overline{\alpha-1}}(t-s+\alpha)^{\overline{\alpha k}} \\
& =\varepsilon \sum_{k=0}^{\infty} \frac{(-\lambda)^{k}}{\Gamma(\alpha k+\alpha)} \sum_{s=0}^{t}(t-s+1)^{\overline{\alpha k+\alpha-1}} \\
& =\varepsilon \sum_{k=0}^{\infty} \frac{(-\lambda)^{k}(t+1)^{\overline{\alpha k+\alpha}}}{\Gamma(\alpha k+\alpha+1)} \\
& =\frac{\varepsilon}{\lambda}\left[1-F_{\alpha}\left(-\lambda,(t+1)^{\bar{\alpha}}\right)\right] \leq \frac{\varepsilon}{\lambda} .
\end{aligned}
$$

Finally, we discuss the Hyers-Ulam stability of the following Caputo type linear fractional nabla difference equation:

$$
\left(\nabla_{0 *}^{\alpha} u\right)(t)+\lambda u(t)=f(t), \quad 0<\alpha<1, \quad \lambda \geq 0 .
$$

Using Definition 2 in (32), we get

$$
\left(\nabla_{0}^{\alpha} u\right)(t)+\lambda u(t)=g(t), \quad t \in \mathbb{N}_{1},
$$

which is similar to (14). Here

$$
g(t)=f(t)+\frac{(t+1)^{-\alpha}}{\Gamma(1-\alpha)} u(0), \quad t \in \mathbb{N}_{0} .
$$

Example 11. Consider the following fractional nabla difference equation:

$$
\left(\nabla_{0}^{0.25} u\right)(t)+(0.09) u(t)=\frac{(t+1)^{\overline{0.25}}}{\Gamma(0.75)}, \quad t \in \mathbb{N}_{1} .
$$

For $\varepsilon=0.1$ and $v(t)=1$ for all $t \in \mathbb{N}_{1}$, we have

$$
\begin{aligned}
& \left|\left(\nabla_{0}^{0.25} v\right)(t)+(0.09) v(t)-\frac{(t+1)^{-0.25}}{\Gamma(0.75)}\right| \\
& \quad=\left|\frac{(t+1)^{-0.25}}{\Gamma(0.75)}+(0.09)-\frac{(t+1)^{-0.25}}{\Gamma(0.75)}\right| \leq \varepsilon .
\end{aligned}
$$

Let $u(0)=1$. Then, the exact solution of (35) is given by

$$
\begin{aligned}
& u(t)=(1.1)(t+1)^{\overline{-0.75}} F_{0.25,0.25}\left(-0.1,(t+0.25)^{\overline{0.25}}\right) \\
& +\sum_{s=1}^{t}(t-s+1)^{\overline{0.75}} F_{0.25,0.25}\left(-0.1,(t-s+0.25)^{\overline{0.25}}\right) \\
& \cdot(s+1)^{\overline{-0.25}}, \quad t \in \mathbb{N}_{0} .
\end{aligned}
$$

Consequently, for $t \in \mathbb{N}_{0}$, we get

$$
|u(t)-v(t)| \leq K(\varepsilon),
$$

where

$$
K(\varepsilon)=\frac{\varepsilon}{\lambda}=1.1111 .
$$

Thus, (35) is Hyers-Ulam stable on $\mathbb{N}_{0}$. Furthermore, we illustrate these concepts numerically in Table 1.

Example 12. Consider the following fractional nabla difference equation:

$$
\begin{aligned}
&\left(\nabla_{0}^{0.75} u\right)(t)+(0.5) u(t)=(0.25)\left(\frac{1}{t+1}\right)^{0.5}, \\
& t \in \mathbb{N}_{1} .
\end{aligned}
$$

For $v(t)=(t+1)^{\overline{0.25}}$, we have

$$
\begin{aligned}
& \left|\left(\nabla_{0}^{0.75} u\right)(t)+(0.5) u(t)-(0.25)\left(\frac{1}{t+1}\right)^{0.5}\right| \\
& \quad=\mid \frac{\Gamma(1.25)}{\Gamma(0.75)}(t+1)^{\overline{-0.5}}+(0.5)(t+1)^{\overline{0.25}} \\
& \quad-(0.25)\left(\frac{1}{t+1}\right)^{0.5} \mid \leq \frac{\Gamma(1.25)}{\Gamma(0.75)}(t+1)^{-0.5}+(0.5)(t \\
& \quad+1)^{\overline{0.25}}+(0.25)\left(\frac{1}{t+1}\right)^{0.5} \leq 0.7397(t+1)^{\overline{-0.5}} \\
& \quad+(0.5)(t+1)^{\overline{0.25}}+(0.25)(t+1)^{\overline{-0.5}}=(t+1)^{\overline{-0.5}} \\
& \quad+(0.5)(t+1)^{\overline{0.25}}=\phi(t), \quad t \in \mathbb{N}_{0} .
\end{aligned}
$$




\begin{tabular}{ccccccc}
\multicolumn{7}{c}{ TABLE 1 } \\
\hline$t$ & $v(t)$ & $|w(t)|$ & $\varepsilon$ & $u(t)$ & $|u(t)-v(t)|$ & $K(\varepsilon)$ \\
\hline 0 & 1 & 0.09 & 0.1 & 1.0000 & 0 & 1.1111 \\
1 & 1 & 0.09 & 0.1 & 1.1364 & 0.1364 & 1.1111 \\
2 & 1 & 0.09 & 0.1 & 1.1389 & 0.1389 & 1.1111 \\
3 & 1 & 0.09 & 0.1 & 1.1346 & 0.1346 & 1.1111 \\
4 & 1 & 0.09 & 0.1 & 1.1292 & 0.1292 & 1.1111 \\
5 & 1 & 0.09 & 0.1 & 1.1238 & 0.1238 & 1.1111 \\
6 & 1 & 0.09 & 0.1 & 1.1188 & 0.1188 & 1.1111 \\
7 & 1 & 0.09 & 0.1 & 1.1140 & 0.1140 & 1.1111 \\
8 & 1 & 0.09 & 0.1 & 1.1096 & 0.1096 & 1.1111 \\
9 & 1 & 0.09 & 0.1 & 1.1054 & 0.1054 & 1.1111 \\
10 & 1 & 0.09 & 0.1 & 1.1015 & 0.1015 & 1.1111 \\
11 & 1 & 0.09 & 0.1 & 1.0979 & 0.0979 & 1.1111 \\
12 & 1 & 0.09 & 0.1 & 1.0944 & 0.0944 & 1.1111 \\
\hline
\end{tabular}

TABLE 2

\begin{tabular}{ccccccc}
\hline$t$ & $v(t)$ & $|w(t)|$ & $\phi(t)$ & $u(t)$ & $|u(t)-v(t)|$ & $\psi(t)$ \\
\hline 0 & 0.9064 & 1.5142 & 2.2257 & 0.9064 & 0 & 0.0362 \\
1 & 1.1330 & 1.0452 & 1.4527 & 0.5711 & 0.5619 & 1.4980 \\
2 & 1.2746 & 0.9846 & 1.3020 & 0.4384 & 0.8362 & 2.4424 \\
3 & 1.3808 & 0.9751 & 1.2443 & 0.3618 & 1.0190 & 3.2293 \\
4 & 1.4672 & 0.9803 & 1.2182 & 0.3110 & 1.1562 & 3.9384 \\
5 & 1.5405 & 0.9908 & 1.2064 & 0.2746 & 1.2659 & 4.6007 \\
6 & 1.6047 & 1.0036 & 1.2045 & 0.2471 & 1.3576 & 5.2317 \\
7 & 1.6620 & 1.0172 & 1.2023 & 0.2257 & 1.4363 & 5.8401 \\
8 & 1.7139 & 1.0311 & 1.2050 & 0.2084 & 1.5055 & 6.4313 \\
9 & 1.7616 & 1.0449 & 1.2095 & 0.1942 & 1.5674 & 7.0092 \\
10 & 1.8056 & 1.0584 & 1.2151 & 0.1824 & 1.6232 & 7.5762 \\
11 & 1.8466 & 1.0716 & 1.2214 & 0.1723 & 1.6743 & 8.1343 \\
12 & 1.8851 & 1.0845 & 1.2282 & 0.1636 & 1.7215 & 8.6849 \\
\hline
\end{tabular}

Let $u(0)=\Gamma(1.25)$. Then, the exact solution of (40) is given by

$$
\begin{aligned}
& u(t)=(1.5) \Gamma(1.25)(t+1)^{\overline{-0.25}} \\
& \cdot F_{0.75,0.75}\left(-0.5,(t+0.75)^{\overline{0.75}}\right)+(0.25) \\
& \quad \cdot \sum_{s=1}^{t}(t-s+1)^{\overline{-0.25}} F_{0.75,0.75}\left(-0.5,(t-s+0.75)^{\overline{0.75}}\right) \\
& \quad \cdot\left(\frac{1}{s+1}\right)^{0.5}, \quad t \in \mathbb{N}_{0} .
\end{aligned}
$$

Consequently, we get

$$
|u(t)-v(t)| \leq \psi(t),
$$

where

$$
\begin{aligned}
\psi(t) & =\nabla_{1}^{-0.75} \phi(t)=\nabla_{0}^{-0.75} \phi(t)-\frac{(t+1)^{\overline{-0.25}}}{\Gamma(0.75)} \phi(0) \\
& =\frac{\Gamma(0.5)}{\Gamma(1.25)}(t+1)^{\overline{0.25}}+(0.5) \frac{\Gamma(1.25)}{\Gamma(2)}(t+1)^{\overline{1}}
\end{aligned}
$$

$$
\begin{aligned}
& -\frac{(t+1)^{\overline{-0.25}}}{\Gamma(0.75)} \phi(0) \\
= & (1.9954)(t+1)^{\overline{0.25}}+(0.4532)(t+1) \\
& -(1.8162)(t+1)^{\overline{-0.25}}, \quad t \in \mathbb{N}_{0} .
\end{aligned}
$$

Thus, (40) is generalized Hyers-Ulam stable. Furthermore, we illustrate these concepts numerically in Table 2.

\section{Competing Interests}

The author declares that they have no competing interests.

\section{References}

[1] S. M. Ulam, A Collection of Mathematical Problems, Interscience Tracts in Pure and Applied Mathematics no. 8, Interscience, New York, NY, USA, 1960.

[2] D. H. Hyers, "On the stability of the linear functional equation," Proceedings of the National Academy of Sciences of the United States of America, vol. 27, pp. 222-224, 1941.

[3] T. M. Rassias, "On the stability of the linear mapping in Banach spaces," Proceedings of the American Mathematical Society, vol. 72, no. 2, pp. 297-300, 1978.

[4] S.-M. Jung, Hyers-Ulam-Rassias Stability of Functional Equations in Nonlinear Analysis, Springer, New York, NY, USA, 2011.

[5] P. L. Kannappan, Functional Equations and Inequalities with Applications, Springer Monographs in Mathematics, Springer, Berline, Germany, 2009.

[6] H. Rezaei, S.-M. Jung, and T. M. Rassias, "Laplace transform and Hyers-Ulam stability of linear differential equations," Journal of Mathematical Analysis and Applications, vol. 403, no. 1, pp. 244251, 2013.

[7] C. Wang and T.-Z. Xu, "Hyers-Ulam stability of a class of fractional linear differential equations," Kodai Mathematical Journal, vol. 38, no. 3, pp. 510-520, 2015.

[8] C. Wang and T.-Z. Xu, "Hyers-Ulam stability of fractional linear differential equations involving Caputo fractional derivatives," Applications of Mathematics, vol. 60, no. 4, pp. 383-393, 2015.

[9] J. R. Wang and X. Li, "A uniform method to Ulam-Hyers stability for some linear fractional equations," Mediterranean Journal of Mathematics, vol. 13, no. 2, pp. 625-635, 2016.

[10] C. Goodrich and A. C. Peterson, Discrete Fractional Calculus, Springer International, New York, NY, USA, 2015.

[11] T. Abdeljawad, "On Riemann and Caputo fractional differences," Computers \& Mathematics with Applications, vol. 62, no. 3, pp. 1602-1611, 2011.

[12] A. Nagai, "Discrete Mittag-Leffler function and its applications," Publications of the Research Institute for Mathematical Sciences, Kyoto University, vol. 1302, pp. 1-20, 2003.

[13] F. M. Atici and P. W. Eloe, "Linear systems of fractional nabla difference equations," Rocky Mountain Journal of Mathematics, vol. 41, no. 2, pp. 353-370, 2011. 


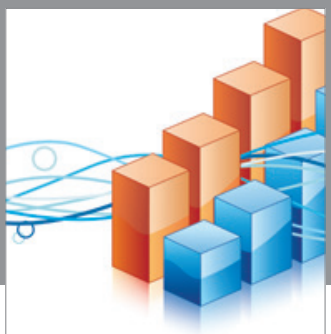

Advances in

Operations Research

vatem alat4

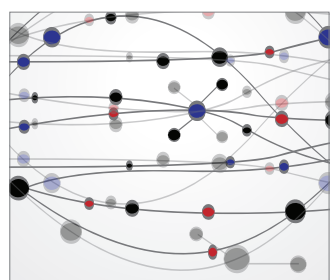

\section{The Scientific} World Journal
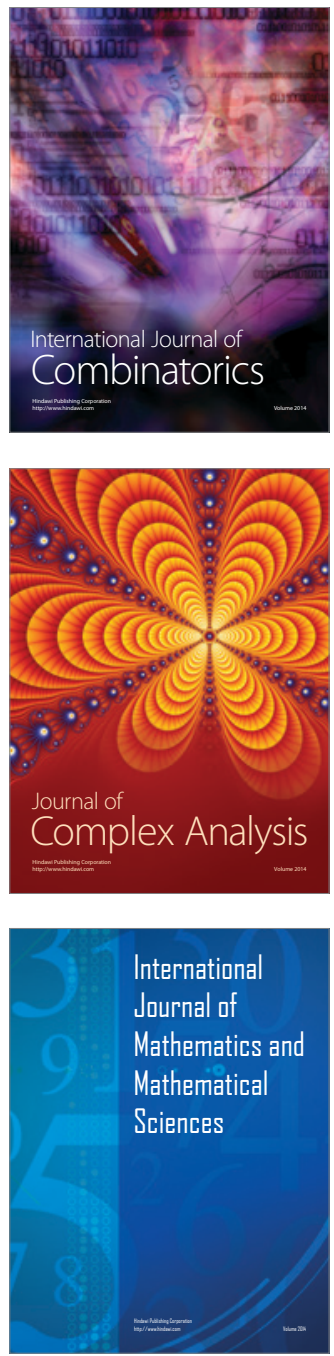
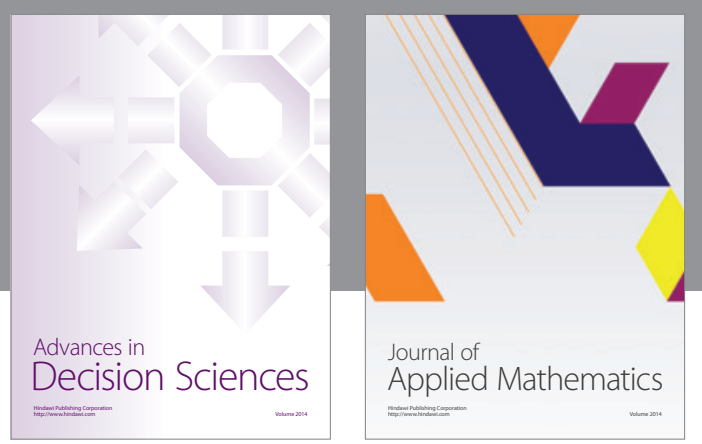

Algebra

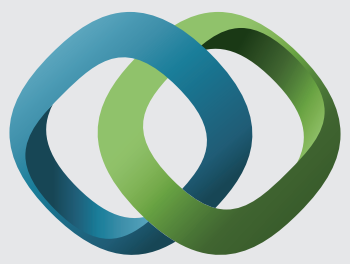

\section{Hindawi}

Submit your manuscripts at

http://www.hindawi.com
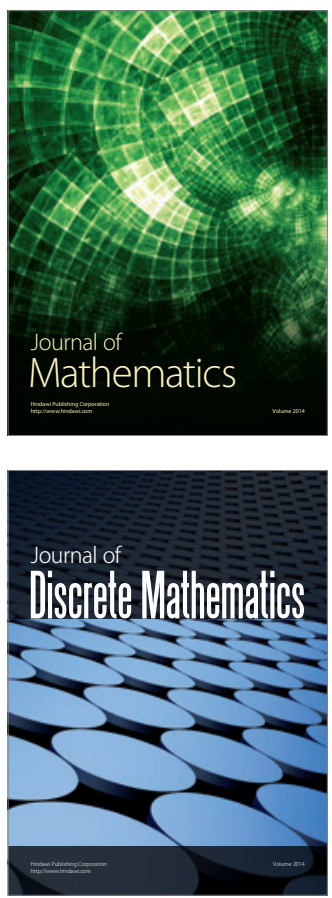

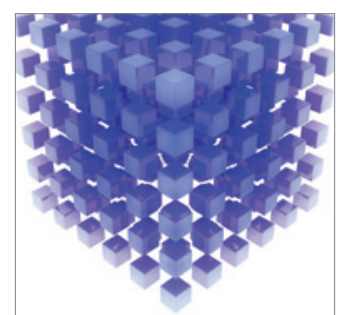

Mathematical Problems in Engineering
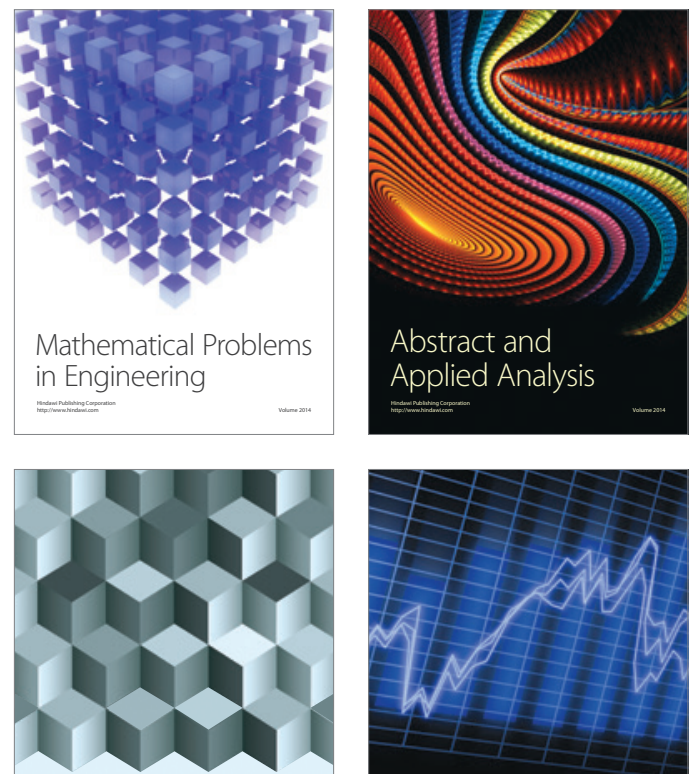

Journal of

Function Spaces

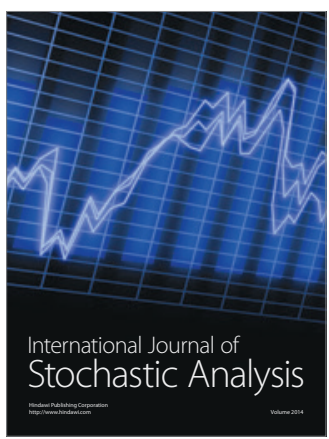

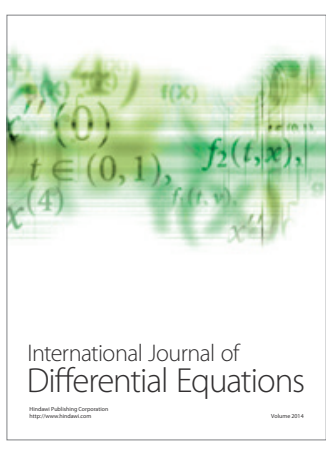
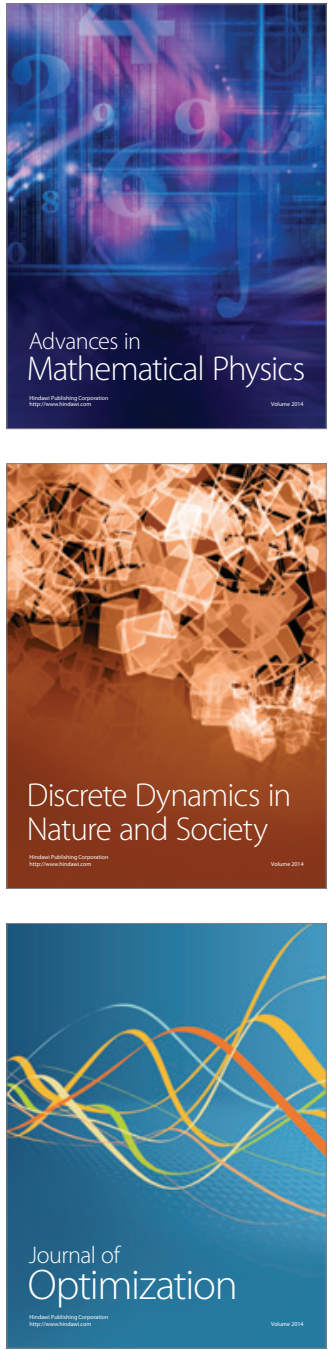